\title{
Efectividad de una intervención educativa con prescripción enfermera para el control del fósforo en pacientes en hemodiálisis
}

\author{
Laia Reixach-Aumatell, Maria Cufí-Vallmajor, Esther Martín-Rabassa, Alicia Rey-Miguel \\ Servicio de Nefrología. Fundació Salut Empordà. Hospital de Figueres. Girona. España
}

Como citar este artículo:

Reixach-Aumatell L, Cufí-Vallmajor M, Martín-Rabassa E, Rey-Miguel A. Efectividad de una intervención educativa con prescripción enfermera para el control del fósforo en pacientes en hemodiálisis. Enferm Nefrol. 2021 Jul-Sep;24(3):304-12

Introducción: El control del fósforo en pacientes en hemodiálisis es muy importante para la prevención de complicaciones.

Objetivo principal: Evaluar los niveles de fósforo en sangre antes, durante y después de las intervenciones médica y enfermera-médica.

Material y Método: Estudio cuantitativo, analítico, cuasi experimental, longitudinal y prospectivo en una población de pacientes en hemodiálisis hospitalaria. El estudio tuvo una duración de 18 meses donde enfermeras y médicos en diferentes meses controlaron la prescripción de los quelantes del fósforo. Se recogieron datos de los resultados analíticos y se reforzó la pauta dietética por parte de las enfermeras. Se administró el test de Morisky Green para valorar la adherencia al tratamiento, así como un "check list" para valorar los efectos secundarios de los quelantes.

Resultados: Se estudiaron 19 pacientes. Los niveles de fósforo (mediana; rango intercuartílico) en los distintos periodos fueron de: $(4,8 ; 1,3) \mathrm{mg} / \mathrm{dl}$ en el periodo previo, $(4,5 ; 1,9) \mathrm{mg} / \mathrm{dl}$ en el periodo médico y $(4,5$; $0,9 \mathrm{mg} / \mathrm{dl}$ ) en el periodo enfermera-médico. Se compararon los resultados entre los diferentes periodos y no se observaron diferencias estadísticamente significativas entre los niveles de fósforo. Tampoco se observaron

\section{Correspondencia:}

Laia Reixach Aumatell

E-mail: Ireixach2@salutemporda.cat diferencias en dichos niveles al separar los pacientes cumplidores de los no cumplidores.

Conclusiones: Aunque los resultados no fueron estadísticamente diferentes, se observó una disminución del fósforo con el refuerzo de dieta continuo por parte de las enfermeras.

Por lo que se concluye que las enfermeras, junto con el apoyo médico, podrían implicarse en la prescripción y el control de fósforo mensual de los pacientes de la Unidad.

PALABRAS CLAVE: fósforo sérico; hemodiálisis; dieta; enfermera; cumplimiento de la medicación.

\section{Effectiveness of a nurse-prescribed educational intervention for phosphorus control in hemodialysis patients}

Introduction: Phosphorus control in hemodialysis patients is very important for the prevention of complications.

Objective: To assess blood phosphorus levels before, during and after medical and nurse-medical interventions.

Material and Method: Quasi-experimental, analytical, longitudinal and prospective study in a population of hospital hemodialysis patients. The study lasted 18 months in which nurses and doctors monitored the prescription of phosphorus chelators during different months. Data on analytical results were collected 
and dietary guidelines were reinforced by the nurses. The Morisky Green test was administered to assess adherence to treatment, as well as a checklist to assess the side effects of the chelators.

Results: A total of 19 patients were studied. The phosphorus levels (median; interquartile range) were: $(4.8 ; 1.3) \mathrm{mg} / \mathrm{dl}$ in the previous period, $(4.5 ; 1.9) \mathrm{mg} / \mathrm{dl}$ in the physician period and $(4.5 ; 0.9 \mathrm{mg} / \mathrm{dl})$ in the nursephysician period. The results were compared between the different periods and no statistically significant differences were observed between the phosphorus levels. Nor were differences in these levels observed when separating compliant and non-compliant patients.

Conclusions: Although no significant differences were found, a decrease in phosphorus was observed with continuous dietary reinforcement by nurses. It is therefore concluded that nurses, together with medical support, could be involved in the monthly phosphorus prescription and control of the unit's patients.

KEYWORDS: serum phosphorus; dialysis; diet; nurses; medication adherence.

\section{Introducción}

La escasa adherencia al tratamiento farmacológico es un problema importante entre los pacientes con Insuficiencia Renal Crónica (IRC) y requiere una actuación específica. De manera general, se estima que la falta de adherencia en los pacientes con enfermedades crónicas llega al $45 \%^{1,2}$.

En general, en los tratamientos de enfermedades crónicas se va perdiendo adherencia con el transcurso del tiempo y hace que se complique seriamente la evolución de la enfermedad por distintas razones ${ }^{3}$.

El incumplimiento terapéutico o falta de adherencia al tratamiento farmacológico es un problema prevalente y relevante en la práctica clínica, que tiende a aumentar y es especialmente frecuente en las enfermedades crónicas. Estudios previos han descrito que la falta de adherencia al tratamiento en enfermedades crónicas oscila, según la patología, entre el 30-60\%. Los pacientes en hemodiálisis no son ajenos a este problema pues necesitan una amplia terapia farmacológica que complemente a la diálisis ya que sus niveles de fósforo se ven aumentados, descienden las cifras de calcio, la producción de hormonas como la Eritropoyetina se ven disminuidas, así como la formación de hematíes y de Vitamina D activa influyendo en la mineralización ósea².

Se considera que los valores de fósforo normales deben oscilar entre 2,3-4,7 mg/dl. Cuando los riñones funcionan con normalidad eliminan el fósforo de la sangre, pero cuando se sufre IRC, ya desde estadios iniciales, los riñones no pueden eliminar el fósforo de forma correcta provocando concentraciones elevadas en sangre que son perjudiciales para el organismo ${ }^{4}$.

Una concentración elevada de calcio y fósforo puede producir depósitos peligrosos de calcio en los vasos sanguíneos, pulmones, ojos y corazón. El control de estos minerales es muy importante para prevenir la progresión de calcificaciones vasculares y otras complicaciones que son un importante factor de riesgo de mortalidad $^{5}$.

Los niveles de fósforo elevados en estos pacientes se pueden controlar con restricciones en la dieta, farmacológicamente con la reducción de la absorción intestinal a través de quelantes, o mediante la depuración del fósforo con la terapia renal sustitutiva ${ }^{5,6}$.

El tratamiento inicialmente será la restricción de alimentos ricos en fósforo, sin comprometer el aporte de proteínas. Se inicia cuando los niveles séricos de la parathormona (PTH) o de fósforo empiezan a elevarse. Las guías K/DOQI recomiendan un aporte de fósforo de 10-12 mg/g proteína, seleccionando aquellos alimentos con una mayor fuente de proteína y menor cantidad de fósforo. Es muy difícil para el personal sanitario y dietistas saber la cantidad de fósforo ingerida durante el día, ya que en las tablas de alimentos no se refleja el fósforo que se encuentra en los aditivos. Por ello se recomienda a los pacientes controlar la ingesta de alimentos procesados y preparados ${ }^{7,8,9}$.

Los quelantes, medicación utilizada para controlar los niveles de fósforo, se administran entre las comidas para reducir la cantidad de $\mathrm{P}$ que absorbe el organismo de los alimentos ingeridos ${ }^{10,11}$.

Teniendo en cuenta estas dificultades descritas en la introducción, en este trabajo se plantearon diferentes abordajes para controlar y mejorar los niveles de fósforo de nuestros pacientes. Como objetivo principal nos planteamos evaluar los niveles de fósforo en sangre antes, durante y después de las intervenciones médica y enfermera-médica. Como objetivos secundarios, se plantearon determinar la eficacia de la educación die- 
tética por parte de las enfermeras a partir del control analítico de niveles de fósforo en sangre; y evaluar el aumento o disminución de los efectos secundarios de los quelantes en los pacientes.

\section{Material y método}

\section{Diseño y participantes}

Se llevó a cabo un estudio cuantitativo, analítico, cuasi experimental, longitudinal y prospectivo realizado entre febrero de 2019 y julio de 2020 en la Unidad de diálisis del Hospital de Figueres.

Se incluyeron las personas con insuficiencia renal crónica terminal que se encontraban en tratamiento renal sustitutivo en la Unidad de Diálisis durante los meses de estudio que cumplían los siguientes criterios de inclusión: ser tratados con hemodiálisis convencional o hemodiafiltración en línea, presentar niveles de fósforo sérico alterados al inicio del estudio $(>4,5 \mathrm{mg} / \mathrm{dl})$, precisar tratamiento farmacológico para el control del fósforo, y haber iniciado hemodiálisis, al menos, seis meses antes del inicio de la intervención médica.

Como criterios de exclusión se determinaron: realizar menos de $12 \mathrm{~h} / \mathrm{semana}$ de tratamiento con hemodiálisis, ser tratado con diálisis peritoneal, presentar alteraciones psíquicas y/o cognitivas, pacientes no colaboradores, con limitaciones educativas y de comprensión del lenguaje escrito; y rechazo del paciente de participar en el estudio.

Como criterios de retirada se establecieron los siguientes: voluntad del paciente de abandonar el estudio; salida del programa de hemodiálisis por trasplante, exitus o cambio de tratamiento.

\section{Periodos de estudio}

- Periodo previo

Se registraron 6 meses sin intervención, solo se entregó la prescripción de medicación pautada por los nefrólogos como habitualmente.

\section{- Periodo médico}

Los nefrólogos realizaron un protocolo para la realización del ajuste de dosificación de quelantes del fósforo, que fue utilizado por todos los profesionales (médicos y enfermeras) durante los periodos en los que se llevó a cabo el estudio. En los siguientes 6 meses de estudio, la prescripción de quelantes a los pacientes la llevaron a cabo los nefrólogos, ajustando las dosis en función de los resultados analíticos y según marcaba el protocolo establecido (ver tabla 1).

\section{- Periodo enfermera-médico}

Los 6 últimos meses, fueron las enfermeras quienes propusieron las dosis de quelantes a prescribir en función de los resultados analíticos y siguiendo el protocolo establecido. Los nefrólogos validaron o modificaron la propuesta de las enfermeras y realizaron la prescripción definitiva. Por parte de las enfermeras se elaboró una hoja de consejo dietético (ver Anexos 1 y 2) con la que se llevaron a cabo dos intervenciones educativas sobre la dieta a todos los pacientes de la muestra: una antes de la intervención médica, y la segunda, antes de la intervención enfermera-médica.

La información contenida en dicha hoja la transmitió enfermería en las dos intervenciones siguiendo una pauta establecida y seguidamente se entregó por escrito una hoja de consejo dietético. En estos meses las enfermeras también realizaron refuerzo dietético a los pacientes en los que no se detectó mejoría del fósforo con la medicación.

\section{Variables a estudio}

- Variables sociodemográficas: edad y sexo.

- Fósforo sérico: considerando como parámetros normales los valores entre 2,5 y $4,5 \mathrm{mg} / \mathrm{dl}$. Se recogieron los valores de los siguientes periodos: seis meses sin intervención, seis meses de periodo médico y seis meses de periodo enfermera-médico. Los valores de fósforo fueron recogidos coincidiendo con los controles analíticos mensuales, que se realizaron de forma protocolizada.

Tabla 1. Resumen protocolo del ajuste de quelantes.

\begin{tabular}{|c|c|c|}
\hline $\begin{array}{l}\text { NIVELES } \\
\text { FÓSFORO }\end{array}$ & AJUSTE QUELANTE & OBSERVACIONES \\
\hline$<2,5 \mathrm{mg} / \mathrm{dl}$ & Disminuir un comprimido al dia & \\
\hline $2,5-4,5 \mathrm{mg} / \mathrm{dl}$ & Mantener dosis & \\
\hline$>4,5 \mathrm{mg} / \mathrm{dl}$ & Augmentar un comprimido al dia & $\begin{array}{c}\text { - Si es la primera determinación } \\
\text { elevada, se realizará refuerzo dietético } \\
\text { y adherencia al tratamiento }\end{array}$ \\
\hline
\end{tabular}


- Albúmina sérica: variable secundaria. Considerando parámetros normales los valores entre 32 y $46 \mathrm{~g} / \mathrm{L}$. Este valor se recogió antes de los tres periodos del estudio.

- Pauta de medicación prescrita en cada periodo: fármaco, dosis y frecuencia.

- Efectos secundarios de los quelantes del fósforo: se registró la presencia de diarrea, estreñimiento, dolor abdominal, dispepsia, náuseas y vómitos.

- Adherencia al tratamiento: determinado mediante el test de Morisky Green a los pacientes al inicio del estudio, del periodo médico y del periodo enfermera-médico. Consideramos paciente incumplidor aquel que responde incorrectamente a 10 más de las 4 preguntas del cuestionario. (Ver anexo 3 ).

\section{Recogida de datos}

Las variables a estudio fueron recogidas por los miembros del equipo multidisciplinar en una hoja de registro diseñada ad hoc.

\section{Aspectos éticos}

El estudio se presentó para su aprobación al Comité Ético de Investigación Clínica (CEIC) Girona para certificar que se garantizaban todos los aspectos éticos y se salvaguardaba la confidencialidad de los pacientes participantes, en el que se obtuvo un dictamen favorable. Los pacientes que formaron parte del estudio se mantuvo la confidencialidad según la Ley Orgánica 3/2018, de 5 de diciembre, de Protección de Datos Personales y garantía de los derechos digitales (LOPDGDD).

El estudio cumple con el Reglamento (UE) 2016/679 del Parlamento Europeo y del Consejo de 27 de Abril de 2016 relativo a la protección de las personas físicas por lo que se refiere al tratamiento de datos personales y la libre circulación de esos datos y por el cual se deroga la Directiva 95/46/CE (Reglamento General de Protección de Datos); se informó a los pacientes de forma verbal y escrita, y después de resolver cualquiera de sus dudas se les pidió que firmaran el consentimiento informado para poder participar en el estudio de manera voluntaria.

El estudio se efectuó de acuerdo con los Principios Éticos establecidos en la versión más reciente de la Declaración de Helsinki (18 ${ }^{\text {th }}$ World Medical Assembly, 1964) o las Normas de buena Practica uti- lizadas en clínica, siempre con la norma que ofrezca más protección al paciente.

\section{Análisis estadístico}

A partir de los datos recogidos se elaboró una base de datos tipo Excel. Para el análisis de los datos se utilizó el paquete estadístico SPSS Versión 26.0 Se realizó un análisis descriptivo de los datos, presentando las variables cuantitativas como medianas y rango intercuartilico (RIC) y las variables cualitativas como frecuencias absolutas y relativas. Se compararon los niveles séricos de fósforo entre los tres periodos de estudio, utilizando el test de Fierdman. Se consideró como significativo un nivel crítico observado inferior al $5 \%(p \leq 0,05)$.

\section{Resultados}

La mediana de edad de los pacientes del estudio era de 69 años (RIC:24), siendo 11 (61,11\%) hombres.

El nivel de albúmina de los pacientes, en todos los periodos, se encontraba dentro del rango establecido como normal. En el periodo previo la mediana de albúmina fue de $36 \mathrm{~g} / \mathrm{L}$ (RIC:3), en el periodo médico la mediana de albúmina fue de $38 \mathrm{~g} / \mathrm{L}$ (RIC:6) y en periodo enfermera-médico la mediana de albúmina se encontró en 39g/L (RIC:3).

De los 19 pacientes del estudio, se determinó el nivel de fósforo los seis meses previos al inicio del estudio, seis meses durante la intervención médica y seis meses durante la intervención enfermera-médico.

Los resultados del test Morkisky-Green mostraron que los pacientes no cumplidores fueron los mismos en los tres periodos. En la tabla 2 se puede apreciar los niveles medianos mensuales de fósforo de los pacientes cumplidores, no cumplidores y total de pacien-

Tabla 2. Comparación de los valores de fósforo serico durante los tres periodos de estudio según la adherencia al tratamiento.

\begin{tabular}{|l|c|c|c|}
\hline \multirow{2}{*}{} & \multicolumn{4}{|c}{ Niveles de fósforo mg/dl (Mediana;RIC) } \\
\cline { 2 - 5 } & Periodo previo & Periodo médico & Periodo enfermera \\
\hline Cumplidores $(n=14)$ & $4,55($ RIC:1,12) & $4,40($ RIC:1,10) & $4,40($ RIC:1,47) \\
\hline No cumplidores $(n=5)$ & $5,60($ RIC:2,1) & $4,50($ RIC:3,2) & $4,40($ RIC:1,2) \\
\hline Muestra total $(n=19)$ & $4,80($ RIC:1,3) & $4,50($ RIC:1,9) & $4,50($ RIC:0,9) \\
\hline
\end{tabular}


Tabla 3. Efectos secundarios manifestados por los pacientes durante los 3 periodos de estudio.

\begin{tabular}{|c|c|c|c|c|c|}
\hline \multirow{3}{*}{$\begin{array}{l}\text { Efecto } \\
\text { secundario }\end{array}$} & \multicolumn{5}{|c|}{ Periodo } \\
\hline & \multicolumn{2}{|c|}{$\begin{array}{l}\text { Periodo previo } \\
(n=11)\end{array}$} & \multicolumn{2}{|c|}{$\begin{array}{c}\text { Periodo médico } \\
(n=7)\end{array}$} & $\begin{array}{l}\text { enfermera-médico } \\
(n=8)\end{array}$ \\
\hline & $\%$ total & N total efectos & $\%$ total & N total efectos & N total efectos \\
\hline Diarrea & 6 & 31,57 & 5 & 26,31 & 5 \\
\hline Estreñimiento & 4 & 21,05 & 2 & 10,52 & 3 \\
\hline Dolor abdominal & 4 & 21,05 & 2 & 10,52 & 2 \\
\hline Dispepsia & 1 & 5,26 & 2 & 10,52 & 1 \\
\hline Náuseas & 2 & 10,52 & 1 & 5,26 & 1 \\
\hline Vómitos & 1 & 5,26 & 0 & 0 & 0 \\
\hline
\end{tabular}

tes, comparándolos entre los tres periodos de estudio (periodo previo, periodo médico y periodo enfermera).

Los efectos secundarios de los quelantes del fósforo presentados por los pacientes durante los 3 periodos de estudio se muestran en la tabla 3. Los efectos secundarios más comunes en los pacientes fueron diarrea, estreñimiento y dolor abdominal. Un mismo paciente pudo presentar más de un efecto secundario.

\section{Discusión}

El seguimiento farmacoterapéutico y la educación sanitaria mejoran la adherencia al tratamiento $0^{5}$ y los niveles de fósforo para, a la larga, prevenir la progresión de calcificaciones vasculares ${ }^{7,8}$ y otros factores de riesgo de mortalidad.

En el presente estudio se observó que, incidiendo dos veces al año sobre el factor dieta, se reducen los niveles séricos de fósforo, aunque los resultados no fueron estadísticamente significativos; no obstante la tendencia al descenso de los valores séricos de fósforo muestran la importancia del refuerzo dietético por parte de enfermería.

En este estudio solo se tuvo en consideración a los pacientes con fósforo elevado por encima de $4,5 \mathrm{mg} / \mathrm{dl}$ para realizar la intervención dietética por parte de las enfermeras mientras que en otros estudios, hemos visto, que consideran necesaria esta acción en todos los pacientes de la unidad para evitar el empeoramiento más futuro ${ }^{12}$.

En nuestro estudio, el porcentaje de no cumplidores es bajo, ya que contamos con el $26,31 \%$ de pacientes no cumplidores y en algunos estudios la falta de adherencia al tratamiento oscila entre $30-60 \%{ }^{13}$.

Para poder mejorar aún más esa adherencia al tratamiento se podría incluir la educación dietética no sólo a los pacientes, sino también a los cuidadores principales de los pacientes de edad avanzada ya que son ellos los que les preparan las comidas a diario, así como la preparación y administración de medicación ${ }^{14}$.

La realización de actividades educativas sobre alimentación y cumplimentación farmacológica podría ser útil para disminuir la toma de quelantes de fósforo, reduciendo así el número de pastillas que toman los pacientes; debemos tener en cuenta que a mayor número de fármacos prescritos, menor es la adherencia al tratamiento ${ }^{15}$.

La literatura nos muestra que en los tratamientos de enfermedades renales crónicas se va perdiendo adherencia con el transcurso del tiempo ${ }^{3}$, es por eso que se ha querido evaluar si con una mayor implicación enfermera ante el cumplimento del tratamiento medicamentoso, se conseguían disminuir los niveles séricos de fósforo, incluso en el paciente no cumplidor.

Aunque los estudios muestran que la disminución de fósforo es mayor en los pacientes cumplidores que en los no cumplidores ${ }^{16}$, en nuestro estudio, si separamos el grupo de pacientes en cumplidores y no cumplidores los resultados siguen sin alcanzar la significación estadística en las distintas intervenciones. Observamos una disminución de dichos niveles posteriores a la intervención médico-enfermera. Si nos fijamos, concretamente, en los niveles de fósforo del grupo de no cumplidores, observamos una reducción de $0,64 \mathrm{mg} / \mathrm{dl}$ respecto los resultados de la media de la 
intervención previa y la media de la intervención enfermera-médico, valores que pueden ser clínicamente relevantes. Aun así, hay pacientes que a pesar del refuerzo dietético y medicamentoso siguen sin cumplir la pauta establecida para su tratamiento.

Aunque los resultados de los niveles de fósforo no son estadísticamente significativos entre las diferentes intervenciones, se puede observar una disminución de dichos niveles posteriores a la intervención médico-enfermera que podrían ser clínicamente relevantes.

Al analizar las medias de fósforo en los tres periodos globalmente y cuando los separamos en cumplidores y no cumplidores, observamos una tendencia a la disminución del valor cuando se repasa la medicación que toman en casa y se insiste en la dieta mensualmente de manera individual por parte de las enfermeras.

En cuanto a los efectos secundarios podemos observar que casi no varían entre periodos, ya que los pacientes que están dentro del estudio siguen tomando quelantes, que producen los efectos secundarios mencionados anteriormente.

No hemos encontrado referencias que contemplen la dosificación de quelantes o cualquier otro medicamento en las unidades de diálisis, por lo que no nos ha sido posible la comparación de resultados. Consideramos importante el inicio de estudios del papel de la enfermera en el ámbito de la prescripción y la dosificación de medicación dada la inminente entrada en vigor del Real Decreto 1302/2018, de 22 de octubre por el que se regula la indicación, uso y autorización de dispensación de medicamentos y productos sanitarios de uso humano por parte de los enfermeros.

Como principales limitaciones cabe destacar que sería complicado extrapolar los resultados a otra población, debido a la escasa muestra de pacientes, ya que el estudio se lleva a cabo en un hospital comarcal.

Según la ley vigente de la prescripción enfermera 1302/2018, la prescripción colaborativa la realiza el enfermero en colaboración con un prescriptor independiente. Los medicamentos incluidos en este grupo están sujetos a prescripción médica, pudiendo el enfermero ajustar dosis según la evolución del paciente y bajo protocolos consensuados. Por ello, en este estudio se realizó un protocolo interno.

Es por eso también que el periodo donde las enfermeras proponen la pauta de los quelantes se llama periodo enfermera-médico ya que, en este estudio, las enfermeras sólo pueden elaborar una propuesta de prescripción, pero antes de su aplicación, debe ser validada, o modificada cuando precise por los nefrólogos de la unidad.

Otra limitación importante en este estudio se encuentra en el período enfermera-médico, ya que desde marzo de 2020 se decreta confinamiento por COVID-19 y la carga para las enfermeras es mayor en estos meses por lo que dificulta la realización del trabajo.

A partir de los resultados obtenidos se puede apreciar como los niveles de fósforo en sangre antes, durante y después de las intervenciones se mantienen o mejoran levemente.

No se aprecian diferencias en cuanto a los efectos secundarios de los quelantes durante los tres periodos de estudio.

Cabe destacar como las enfermeras, con un protocolo establecido, pueden gestionar cambios de medicación ya prescrita por los médicos sin causar efectos negativos en los pacientes.

Por todo ello consideramos que las enfermeras, junto con el apoyo médico, podrían implicarse en la prescripción y el control de fósforo mensual de los pacientes de la Unidad.

\section{Fortalezas del estudio}

No se encontraron estudios similares ya que se trata de un estudio novedoso puesto que la prescripción enfermera en Cataluña empieza a ser vigente ahora. Hasta el momento las enfermeras no podían prescribir ningún tipo de medicación.

Recepción: 15-07-21

Aceptación: 25-08-21

Publicación: 30-09-21

\section{Bibliografía}

1. Chamorro MÁ, García-Jiménez $E$, Amariles $P$, Chamorro AR, Merino EM, Martínez FM, Dader MJ. Efecto de la actuación farmacéutica en la adherencia del tratamiento farmacológico de pacientes ambulatorios con riesgo cardiovascular (Estudio EMDADER-CV-INCUMPLIMIENTO). Aten Primaria. 2011;43(5):245-53. 
2. Bover Sanjuán J, Navarro-González JF, Arenas MD, Torregrosa JV, Tamargo Menéndez J, de Francisco ALM, et al. Pharmacological interactions of phosphate binders. Nefrologia (Engl Ed). 2018;38(6):573-8.

3. Contreras F, Esguerra G, Espinosa J, Gutiérrez C, Fajardo L. Adhesión al tratamiento en pacientes con insuficiencia renal crónica en tratamiento de hemodiálisis. Univ. Psychol. Bogotá 2006;5(3):487-99.

4. García-Llana H, Remor E, Selgas R. Adherence to treatment, emotional state and quality of life in patients with end-stage renal disease undergoing dialysis. Psicothema 2013;25(1):79-86.

5. Redondo Simón M C, Casuso Jiménez L, Martínez Jiménez I, Rodríguez Puertas J F, Palacios Gómez María E, Cara Sánchez E. La hiperfosfatemia en paciente renal en programa de hemodiálisis. Enferm Nefrol 2015; 18(supl 1):S124.

6. Cheng TY, Tarng DC, Liao YM, Lin PC. Effects of systematic nursing instruction on a low-phosphorus diet, serum phosphorus level and pruritus of patients on haemodialysis. J Clin Nurs. 2017;26(3-4):48594.

7. Barril-Cuadrado G, Puchulu MB, Sánchez-Tomero JA. Tablas de ratio fósforo/proteína de alimentos para población española. Utilidad en la enfermedad renal crónica. Nefrologia. 2013;33(3):362-71.

8. Cvengros JA, Christensen AJ, Lawton WJ. The role of perceived control and preference for control in adherence to a chronic medical regimen. Ann Dehav Med 2004;27(3):155-61.

9. National Kidney Foundation. K/DOQI clinical practice guidelines for bone metabolism and disease in chronic kidney disease. Am J Kidney Dis 2003; 42 (4 supl 3):S1-201.

10. Narita I, Alchi B, Omori K, Sato F, Ajiro J, Saga D, et al. Etiology and prognostic significance of severe uremic pruritus in chronic hemodialysis patients. Kidney Int. 2006;69(9):1626-32.
11. Torregrosa J-V, Bover J, Cannata Andía J, Lorenzo V, de Francisco ALM, Martínez I, et al. Spanish Society of Nephrology recommendations for controlling mineral and bone disorder in chronic kidney disease patients (SEN-MBD). Nefrologia. 2011;31 (supl 1):S3-32.

12. Díaz Martínez MJ, González Suárez ME, González Suárez E, Fernández Merayo C. Efectividad de las intervenciones de enfermería en el control del fósforo. Enferm Nefrol. 2012;15(supl 1):S110.

13. Ruiz García E, Latorre López LI, Delgado Ramírez A, Crespo Montero R, Sánchez Laguna JL. Adherencia al tratamiento farmacológico en pacientes en hemodiálisis. Enferm Nefrol. 2016;19(3):232-41.

14. Hernández Moreno L, Perinango Romy J, Casanovas Izquierdo E. Influencia de la educación para el control del fósforo sérico realizada por enfermería a pacientes y a sus cuidadores principales. Enferm Nefrol. 2014;17(2):92-7.

15. Neri L, Martini A, Andreucci VE, Gallieni M, Rey LA, Brancaccio D; MigliorDialisi Study Group. Regimen complexity and prescription adherence in dialysis patients. Am J Nephrol. 2011;34(1):71-6.

16. Arenas MD, Pérez-García R, Bennouna M, Blanco A, Mauricio-Reatiga 0, Prados MD et al. Mejoría del cumplimiento terapéutico en pacientes en hemodiálisis con mal control del fósforo y mala adherencia al tratamiento con captores: Estudio COMQUELFOS. Nefrología 2013;33(2):196-203. 


\section{Anexo 1. HOJA DE DIETA PARA LOS PACIENTES}

\section{Consejos para reducir el consumo de fósforo}

\section{CONTROLAR}

Lácteos: 1 ración al día

Yema de huevo:2 a 3 yemas a la semana

Carne y pescado: controlar cantidad

\section{EVITAR}

Productos desnatados

Batidos con chocolate Leche en polvo Quesos semicurados y curados fiambres y embutidos

Precocinados, bollería, pastelería y golosinas Refrescos con burbujas
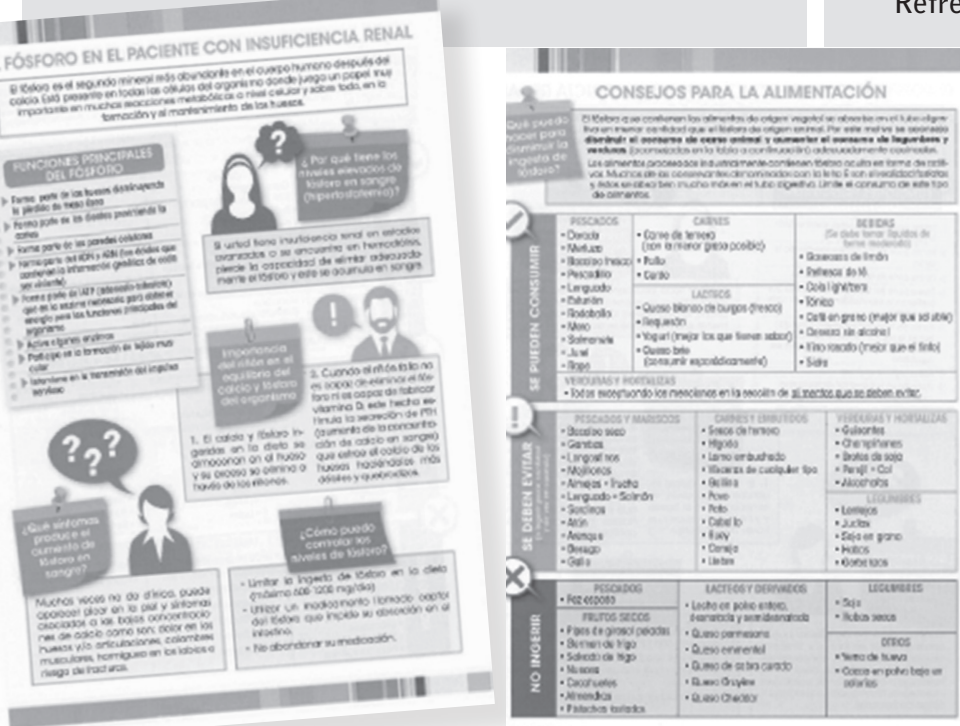

\section{Anexo 2. PAUTA; CONSEJO DIETÉTICO PARA ENFERMERÍA}

\section{PUNTOS IMPORTANTES A TRATAR}

Introducción: qué es el fósforo y para qué sirve

Funciones para el organismo

Efectos en la alteración en la concentración

Distribución en los alimentos

Grupos de alimentos a tener en cuenta: (ver hoja paciente)

Enumerar aditivos

Comentar alimentos precocinados 


\section{Anexo 3. CUESTIONARIO VALIDADO DE MORISKY-GREEN}

\begin{tabular}{l|l}
\hline Pregunta & Respuest \\
\hline ¿Deja de tomar alguna vez los medicamentos para tratar su enfermedad? & SI/NO \\
\hline ¿Toma los medicamentos a las horas indicadas? & SI/NO \\
\hline Cuando se encuentra bien, ¿deja de tomar la medicación? & SI/NO \\
\hline Si alguna vez le sienta mal, ¿deja usted de tomarla? & SI/NO
\end{tabular}

* Se considera cumplidor si se responde de forma correcta a las 4 preguntas: no/sí/no/no.

Este artículo se distribuye bajo una Licencia Creative Commons Atribución-NoComercial 4.0 Internacional. https://creativecommons.org/licenses/by-nc/4.0/

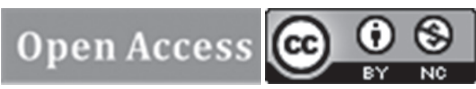

